Proceedings of the 14th International Symposium Ultrafast Phenomena in Semiconductors, Vilnius 2010

\title{
Persistent Currents and Bethe Ansatz
}

\author{
G. KIRŠANSKAS \\ Department of Theoretical Physics, Vilnius University, Sauletekio 9, LT-01108 Vilnius, Lithuania \\ AND A. MATUlis* \\ Semiconductor Physics Institute, Center of Physical Sciences and Technology \\ Goštauto 11, 2600 Vilnius, Lithuania
}

\begin{abstract}
The system of two electrons interacting with a single impurity in 1D metallic ring is considered illustrating the applicability of the Bethe Ansatz.
\end{abstract}

PACS: 73.40.Gk, 03.65.Nk

\section{Introduction}

Persistent current appears in a small metallic ring penetrated by perpendicular magnetic field. This quantum effect is remarkable due to the non-dissipative character of this current. It was predicted by Büttiker [1] in 1983 and later the experimental evidence was obtained by Levy [2]. Due to the progress in nanometer technology there is still interest in persistent currents in nanorings that contain a small numbers of electrons [3]. These currents are rather sensitive to the quantum coherence, and thus to electron interaction and their interaction with impurities, serving as a convenient instrument for studying the subtle properties of strongly interacting electron system. The important role belongs to one-dimensional (1D) models that enable to obtain the analytical solutions and understand the main features of the above phenomena.

The most popular method of analyzing the above systems is the so-called Bethe Ansatz [4] (see also Ref. [5]) based on the assumption that the electron system wave function can be constructed as a superposition of plane waves with the restricted number of wave vectors coordinated with the rules of elastic scattering. For instance, using this Ansatz Li and Ma [6] considered the system of many electrons interacting with an impurity. However, despite numerous applications the motivation of the assumption is still lacking.

The goal of the present work is to illustrate the applicability of the Bethe Ansatz using the most simple model system of two electrons moving in 1D ring and interacting with a single impurity via Dirac function type interaction, and comparing the results with those obtained by means of the exact numerical diagonalization of the Hamiltonian.

\section{Model}

We consider the model system shown in Fig. 1. There are two electrons indicated by full circles moving along

\footnotetext{
* corresponding author; e-mail: amatulis@takas.lt
}

the 1D ring. Impurity is shown by cross. We solve the stationary Schrödinger equation

$$
\{H-E\} \Psi=0,
$$

which is eigenvalue problem for energy and wave function of the system. The Hamiltonian of this equation reads

$$
H=\sum_{n=1}^{2}\left\{\frac{1}{2 m}\left[-\mathrm{i} \hbar \nabla_{n}+\frac{e}{c} \boldsymbol{A}_{n}\right]^{2}+V_{n}\right\}+V_{e e} .
$$

Vector potential $A_{n}=\left[B \times r_{n}\right] / 2$ describes the homogeneous perpendicular magnetic field. Two more symbols $V$ and $V_{e e}$ stand for potentials of the electron interaction with the impurity, and the ee-interaction.

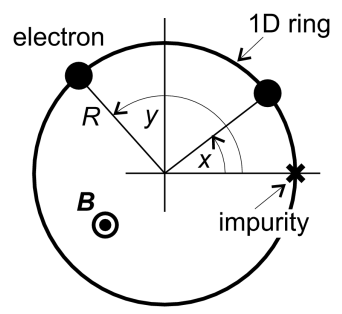

Fig. 1. The layout of the model system.

In order to simplify the model we introduce the dimensionless variables. We shall measure the energy in units $\hbar^{2} / m R^{2}$ and introduce the dimensionless magnetic flux through the ring $\gamma=\pi R^{2} B / \Phi_{0}$ measured in units of the elementary flux $\Phi_{0}=2 \pi \hbar c / e$. Moreover, we assume that all interactions are of the short range, and replace the potentials by the corresponding Dirac functions. Finally, denoting the position of electrons by angles

$$
0 \leqslant x, y \leqslant 2 \pi
$$

we arrive at the following Hamiltonian:

$$
\begin{aligned}
H & =-\frac{1}{2}\left(\frac{\partial}{\partial x}+\mathrm{i} \gamma\right)^{2}-\frac{1}{2}\left(\frac{\partial}{\partial y}+\mathrm{i} \gamma\right)^{2} \\
& +P \delta(x-\pi)+P \delta(y-\pi)+\Lambda \delta(x-y),
\end{aligned}
$$

where the constants $P$ and $\Lambda$ characterize the strengths of electron interaction with the impurity and the ee-interaction, correspondingly. The periodic boundary conditions for the wave function and its first derivatives 
have to be added.

The problem can be further simplified by excluding the magnetic flux from the Hamiltonian that is achieved by the following substitution:

$$
\Psi(x, y)=\mathrm{e}^{-\mathrm{i} \gamma(x+y)} \Phi(x, y) .
$$

Next, we replace the Dirac functions by the corresponding boundary conditions, and thus, replace Schrödinger equation (1) with Hamiltonian (4) by simple Helmholtz equation

$$
\left(\frac{\partial^{2}}{\partial x^{2}}+\frac{\partial^{2}}{\partial y^{2}}+2 E\right) \Phi(x, y)=0
$$

that has to be solved in quadratic region (3). Moreover, we are interested only in singlet states. That is why we assume that the wave function is symmetric $\Psi(y, x)=\Psi(x, y)$ with respect to the interchange of electron coordinates. This reduces the region to the triangular one $(y<x)$ with the following boundary conditions:

$$
\begin{aligned}
& \Phi(x, 0)=\mathrm{e}^{-2 \pi \mathrm{i} \gamma} \Phi(2 \pi, x), \\
& \Phi_{y}(x, 0)-\mathrm{e}^{-\mathrm{i} \gamma} \Phi_{x}(2 \pi, x)=2 P \Phi(x, 0), \\
& \left.\left\{\Phi_{x}(x, y)-\Phi_{y}(x, y)\right\}\right|_{y=x}=\Lambda \Phi(x, x) .
\end{aligned}
$$

\section{Persistent current}

Let us remind the essence of the persistent current and Bethe Ansatz presenting the results for a single electron in the ring when there is no impurity and no ee-interaction $(P=\Lambda=0)$. In this case there is no need to use all above described tricks because due to rotational symmetry of the Hamiltonian the wave function of each electron is just the single exponent

$$
\Psi(x)=\mathrm{e}^{\mathrm{i} k x} .
$$

Inserting it into Schrödinger equation and satisfying the periodic boundary conditions we immediately arrive at the following expression for the electron energy:

$$
E=\frac{1}{2}(\gamma+m)^{2}, \quad m=0, \pm 1, \pm 2, \ldots
$$

Its dependence on magnetic flux is shown in Fig. 2. The

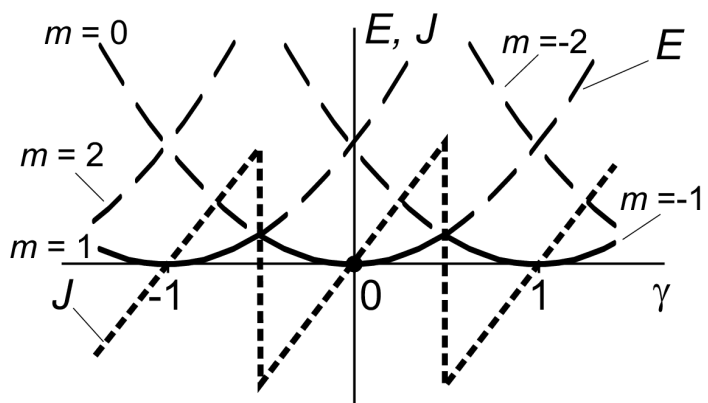

Fig. 2. Single free electron $(P=\Lambda=0)$ in the ring Solid curve - the ground state energy, dotted curve the corresponding persistent current.

ground state energy is shown by solid curve. The dimensionless current (in $c \hbar^{2} / m R^{2} \Phi_{0}$ units) is given as

$$
J=\frac{\mathrm{d} E}{\mathrm{~d} \gamma}=\gamma+m .
$$

The current in the ground state is shown in Fig. 2 by dotted curve. This periodic saw-like curve shows the essence of the persistent current that is non-dissipative and does not vanish even in the ground state.

The elastic electron scattering by the impurity breaks the rotational symmetry, and the electron can change the sign of its orbital momentum $k$ during this scattering event. That is why its wave function has to be chosen as

$$
\Phi(x)=A \mathrm{e}^{\mathrm{i} k x}+B \mathrm{e}^{-\mathrm{i} k x} .
$$

Inserting it into Schrödinger equation and satisfying the boundary conditions we arrive at the following dispersion relation:

$$
\cos (2 \pi \gamma)=\cos (2 \pi k)+\frac{P}{k} \sin (2 \pi k), \quad E=\frac{1}{2} k^{2} .
$$

Its solution - the electron energy dependence on the magnetic flux - is shown in Fig. 3. Now the crossings of different energy levels are changed into anticrossings, and consequently, the persistent current is more soft function, and that is why smaller. We see that the elastic scattering diminishes the persistent current but does not liquidate it.

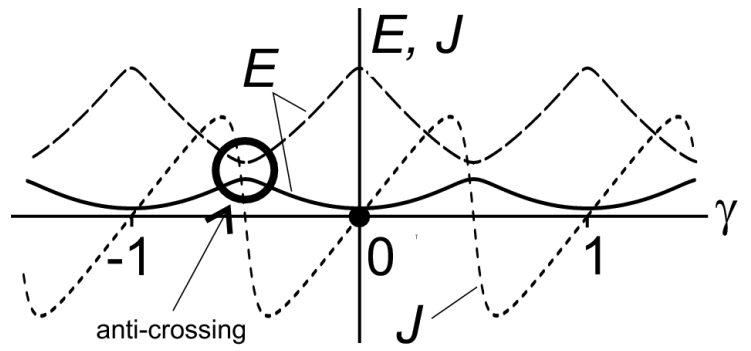

Fig. 3. The same as in Fig. 2, but now $P=1$ and $\Lambda=0$.

By the way, in the case of a single electron in the ring the wave function constructed according to the Bethe Ansatz rules (see Eqs. (8) and (11)) coincides with the exact function following from the solution of the ordinary differential equation.

\section{Two electrons and impurity}

Considering the main problem we denote the orbital momenta of electrons by symbols $k$ and $q$. As the electrons can change the signs of their momenta or interchange them according to the Bethe Ansatz rules we choose the wave function as

$$
\begin{aligned}
& \Phi(x, y)=A \mathrm{e}^{\mathrm{i}(k x+q y)}+B \mathrm{e}^{\mathrm{i}(k x-q y)}+C \mathrm{e}^{\mathrm{i}(-k x+q y)} \\
& +D \mathrm{e}^{-\mathrm{i}(k x+q y)}+a \mathrm{e}^{\mathrm{i}(k y+q x)}+b \mathrm{e}^{\mathrm{i}(k y-q x)} \\
& +c \mathrm{e}^{\mathrm{i}(-k y+q x)}+d \mathrm{e}^{-\mathrm{i}(k y+q x)} .
\end{aligned}
$$

Satisfying boundary conditions (7) and zeroing the coefficients at various exponents we obtain the 12 equations of the following type: 


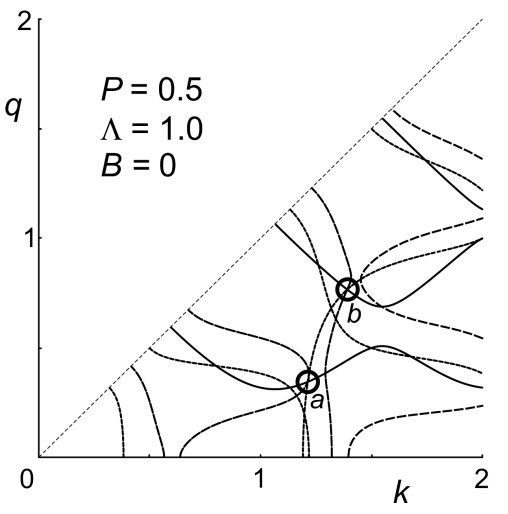

Fig. 4. Graphical solution of the dispersion relations.

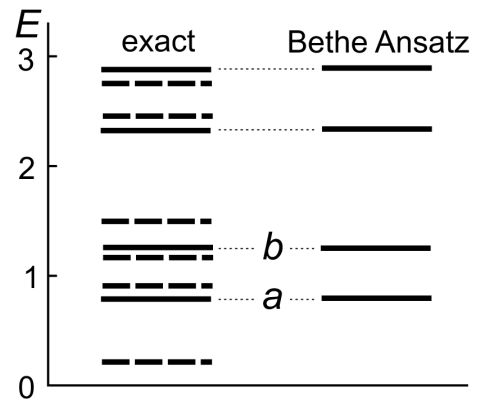

Fig. 5. Energy levels obtained by the Bethe Ansatz and by the exact numerical diagonalization of the Hamiltonian.

$$
A+B-\mathrm{e}^{-2 \pi \mathrm{i} \gamma}\left(\mathrm{e}^{2 \pi \mathrm{i} q} a-\mathrm{e}^{-2 \pi \mathrm{i} q} b\right)=0 .
$$

In general it is not possible to satisfy them having 8 coefficients only. One can obtain the solution only if the equation set is degenerate or reveals some additional symmetry. In order to check this possibility we split the above set into 3 sets with 8 equations in each of them. Zeroing the corresponding determinants we arrived at three dispersion relations $D_{n}(k, q)=0$ with real coefficients. The results of their graphical solution are shown in Fig. 4 as curves (solid, dashed and dotted corresponding to three different relations) in $k q$-plane in the case without magnetic field $(\gamma=0)$. We found the points (two of them

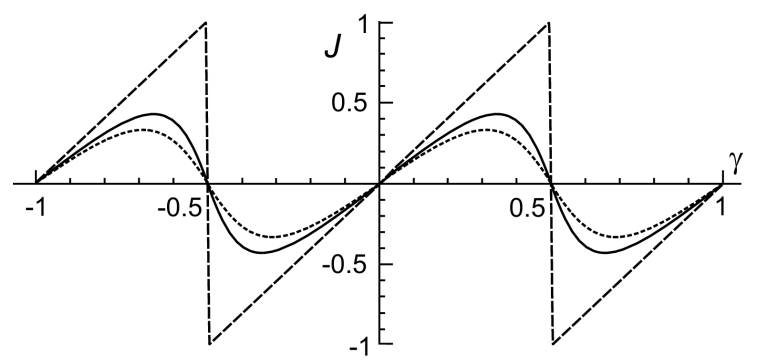

Fig. 6. ee-interaction influence of the persistent current: $P=0, \Lambda=1-$ dashed curve, $P=1, \Lambda=0-$ dotted curve, $P=\Lambda=1-$ solid curve. $a$ and $b$ are shown by circles) where all three curves, corresponding to the simultaneous solution of three dispersion relations, intersect each other. We also checked that the coefficients obtained from these three equation sets coincide as well. Consequently, the above 12 equations have the non-trivial solution, and it seems that the Bethe Ansatz works. In order to make sure of that we have fully solved the problem, and compared this result with exact numerical diagonalization of the considered Helmholtz equation. The comparison of the energy levels obtained by both methods is shown in Fig. 5. We see the coincidence of the energies for all levels obtained using Bethe Ansatz.

However, there are more levels than this Ansatz gives. The reason is in above mentioned overcrowding of the equation set. Inspecting the obtained wave functions we found out that in considered two electron system case the Bethe Ansatz gives only these energy levels whose wave functions are antisymmetric under the reflection of the coordinates over $x+y=2 \pi$ line, namely $\Phi(2 \pi-y$, $2 \pi-x)=-\Phi(x, y)$. As the ground state is symmetric we failed to obtain it. We also failed to obtain the solution via Bethe Ansatz in the case of non zero magnetic field, as the magnetic field breaks the above mentioned symmetry.

Finally, we present the persistent current versus magnetic flux plot in Fig. 6 illustrating the influence of various interactions on it. It is remarkable that the inclusion of the ee-interaction represses the negative role of the impurity scattering. This could be interpreted as a tendency towards Wigner crystallization of this simple model system.

\section{References}

[1] M. Büttiker, Y. Imry, R. Landauer, Phys. Lett. A 96, 365 (1983).

[2] L.P. Levy, G. Dolan, J. Dunsmuir, H. Bouchiat, Phys. Rev. Lett. 64, 2074 (1990).

[3] A.C. Bleszynski-Jayich, W.E. Shanks, B. Peaudecerf, E. Ginossar, F. von Oppen, L. Glazman, J.G.E. Harris, Science 326, 272 (2009).

[4] H. Bethe, Z. Phys. 71, 205 (1931).

[5] M. Karbach, G. Müller, arXiv:cond-mat/9809162, arXiv:cond-mat/9809163, arXiv:cond-mat/0008018.

[6] You-Quan Li, Zhong-Shui Ma, J. Phys. Soc. Jpn. 65, 1519 (1996). 